\title{
FAUNISTIC AND ECOLOGICAL STUDIES ON APIDAE (HYMENOPTERA APOIDEA) IN NATURAL AND CULTIVATED ECOSYSTEMS IN SICILY
}

\author{
a Dipartimento di Agricoltura, Alimentazione e Ambiente, sez. Entomologia applicata. Università degli Studi di Catania, \\ via S. Sofia 100 - 95123 Catania, Italy. \\ "PhD in "Tecnologie Fitosanitarie" - via N. Coviello 26 - 951030 Gravina di Catania (CT), Italy. \\ '(CREA) Consiglio per la ricerca in agricoltura e l'analisi dell'economia agraria. Centro di ricerca olivicoltura, \\ frutticoltura e agrumicoltura. Corso Savoia 190 - 95024 Acireale (CT), Italy.E-mail: salvatore.bella@crea.govit. \\ Corresponding Author: Gaetana Mazzeo,gamazzeo@unict.it
}

Mazzeo G., Longo S., Seminara A.R., Bella S. - Faunistic and ecological studies on Apidae (Hymenoptera Apoidea) in natural and cultivated ecosystems in Sicily.

The authors give the results from more than a decade of studies on Apidae (Hymenoptera, Apoidea) in eastern Sicily, carried out in natural and cultivated ecosystems at different altitudes in the Mediterranean Basal (0-1450 m a.s.l.), and Mountain-Mediterranean plans (1450-2100 m a.s.1.). Seven species are reported for the Mountain-Mediterranean plan at the highest altitude (Piano Provenzana, $1845 \mathrm{~m}$, rich in endemic plants from Etna and Sicily, such as Astragalus, Rubus and Senecio): Anthophora balearica (Friese), A. retusa (L.), Apis mellifera L., Ceratina chalybea Chevrier, Epeolus julliani Peréz, Eucera aterrima Friese and Xylocopa violacea (L.). Thirty-one bee species were recorded in the Mediterranean Basal plan, with Apis mellifera and Xylocopa violacea ubiquitous and abundant in all sites surveyed. Overall, 35 species of Apidae belonging to 11 genera are reported here: Amegilla (2 species), Anthophora (6 species), Apis (1 species), Ceratina (7 species), Epeolus (1 species), Eucera (10 species), Habropoda (1 species), Melecta (1 species), Nomada (3 species), Tetraloniella (1 species), and Xylocopa (2 species). Data on the surveyed species are discussed in relation to the altitudinal level, the plants visited, and the ecological quality of the sites where the specimens were found.

KeY WoRDS: Apidae; Biodiversity; Plants; Ecosystems; Sicily; Altitudinal plans.

\section{INTRODUCTION}

The evaluation of the biodiversity and quality of natural environments and agro-ecosystems is based on the knowledge of the organisms living there, particularly on the study of key species used as bioindicators. Insects are considered good bioindicators, particularly solitary and social bees, due to their relationships to the plants they visit for taking nectar and pollen (PORRINI et al., 2002; Celli and Maccagnani, 2003).

Bees contribute significantly to the pollination of crops and wild plants, and are of great importance for the protection of natural environments (LONGO et al., 2002; Bella, 2014; MARgiotta et al., 2017). In Europe, this group is threatened by habitat loss from agricultural intensification, urban development, and a changing climate. Knowledge about bees and their ecological role is particularly poor in southern European countries. It is therefore essential to analyse recent data about the distribution, population trends, and ecology of the species living in a certain territory that is considered critical for the conservation of bee diversity and for the benefits associated with pollination (NIETO et al., 2014; BALZAN et al., 2016).

In order to know the ecological status of natural and anthropic ecosystems in Sicily, observations were conducted for a period of 11 years on anthophilous insects detected on flowers in different ecosystems. Some of the results regarding crops and some groups of anthophilous insects have already been published, and the data showed that the majority of the insects belonged to Hymenoptera and to the family Apidae (QUARANTA et al., 2004; MAZZEO et al., 2004, 2006, 2007a,b,c; SEMINARA et al., 2009). This paper, is focused on Apidae (Hymenoptera, Apoidea) species recorded in 11 years of surveys, from sea level to the high slopes of the volcano Etna (eastern Sicily). Twelve species (13 taxa) of Bombus Latreille recorded in the same period of investigation, were treated in detail in MAZZEO et al. (2015) and were thus excluded from the discussion.

In this context, this paper represents a first step to the study of ecological traits of bees in a very particular area, the Mount Etna, by providing a list of species, with data on visited plants, altitudinal quotes and features of the areas where the specimens were found.

\section{MATERIALS AND METHODS}

\section{THE INVESTIGATED TERRITORY}

The surveys were carried out from 1997 to 2007 in nine sites representative of the main environments of eastern Sicily, from sea level to $1900 \mathrm{~m}$ a.s.l. on Mount Etna, in Catania province. The sites differed in climate and vegetational aspects. Overall, the climate is Mediterranean, with variations in slope, altitude, and exposure. In the basal plane on the coast, the climate is thermo-Mediterranean (xerothermic-hot), with average temperatures $\geq 16{ }^{\circ} \mathrm{C}$; the following survey sites were located here: Catania (Torre Allegra, and Spina Santa), Giarre (Contrada Nespole), Motta Sant'Anastasì, Paternò and Ramacca. In the hilly zone, the climate is meso-mediterranean with average temperatures between 12 and $16{ }^{\circ} \mathrm{C}$; the following sites were here: Bronte, and Milia (Monte San Leo, Belpasso). In the higher sites of 
the high mountain ranges, the climate is oro-mediterranean with average temperatures $\leq 8^{\circ} \mathrm{C}$ (SIAS, 2014; MAZZEO et al., 2015; Poli Marchese and Patti, 2000); the site of Piano Provenzana (Monte Tanaurpi, Linguaglossa) was here. The vegetational aspects characterising these sites are influenced both by human activity, especially in the mid-baseline plane, and by the activity of the volcano in the central summit. The bioclimatic zones ("altitudinal plans" and "horizons") constituted by plant groupings at various altitudes, include a medium-baseline plan (0-1450 $\mathrm{m}$ a.s.1.) that is characterised by cropland (mainly citrus orchards, olive groves, vineyards, and orchards), oak and pine forests, a mountain plan (1450$2100 \mathrm{~m}$ a.s.1.) that comprise pine and beech forests and pioneer plants in upper altitudinal limit of the arboreal vegetation, a high plan (2100-2950 up to the volcanic desert at $3330 \mathrm{~m}$ a.s.1.) where a characteristic group of species is present. In this area, the predominant plants are Astragalus siculus Biv., capable of colonising the slopes of loose volcanic sand and providing shelter to other species, and some endemic to the volcano, such as Senecio aetnensis Jan ex DC., Viola aetnensis Parl., Anthemis aetnensis Schouw, Rumex aetnensis (C. Pres1), Galium aetnicum Biv., Sclerantus aetnensis Strobl, and Saponaria sicula Rafin. (Poli MarcheSe, 1991).

\section{SAMPLING SITES}

The nine surveyed areas were divided into the following three types based on floristic complexity and the degrees of human and agronomic aspects: agro-ecosystems $[A E]$; natural ecosystems $[N E]$; and semi-natural ecosystems $[S N]$. The sites are described in Table 1.

\section{SAMPLING METHODS}

The protocol of the surveys was developed within the Italian project A.M.A. (Ape, Miele, Ambiente) (QUARANTA et al., 2004) with some modifications. Anthophilous insects were recorded in each site along a transect of 400-600 m, representative of the spontaneous flora that were preliminarily defined in all surveyed areas. The wild plants were collected, prepared and identified in case of doubt by the specialist. The observations were conducted on wild plants once a month, while the cultivated plants were observed three times during the flowering period. The distribution, locality and date of capture, number and sex of examined specimens, number of observed specimens in brackets, and the plants visited are reported for each species. Each specimen was prepared dry and specifically identified after the observation of male sexual structures in cases of doubt.

The classification used in this paper followed MICHENER (2007) for supra-specific taxa, and their nomenclature was according to STOCH (2003) and POLASZEK (2013). Updated distributional data were taken from the online check-list by COMBA (2015), KuHLMANn et al. (2015) and RASMONT and Haubruge (2018). The species were assigned to chorological categories according to LA GRECA (1964).

The collected material is preserved in the collection of the Department of Agriculture, Food and Environment, sect. Applied Entomology, University of Catania.

Acronyms used in the text for the collectors and determiners as follows: AS (Alice Seminara); SB (Salvatore Bella); VL (Vincenzo Lizzio); MQ (Marino Quaranta); MS (Maximilian Schwarz); VN (Vittorio Nobile).

\section{RESULTS}

\section{FAUNISTIC DIVERSITY}

Over the course of 11 years more than 14600 specimens of Apidae were collected or observed, belonging to 35 species.

Table 1 - Sites where the surveys were carried out in 1997-2007.

\begin{tabular}{|c|c|c|c|c|c|}
\hline \multirow{2}{*}{ Sites } & \multirow{2}{*}{ m a.s.l. } & \multirow{2}{*}{$\begin{array}{c}\text { Ecological } \\
\text { types }\end{array}$} & \multirow{2}{*}{$\begin{array}{l}\text { Geographic } \\
\text { coordinates }\end{array}$} & \multicolumn{2}{|r|}{ Description } \\
\hline & & & & General info & Crops and plants \\
\hline Torre Allegra & 5 & {$[S N]$} & $37^{\circ} 24^{\prime} \mathrm{N} 15^{\circ} 04^{\prime} \mathrm{E}$ & $\begin{array}{l}\text { Within the nature reserve } \\
\text { "Oasi del Simeto", behind } \\
\text { the coastline }\end{array}$ & $\begin{array}{l}\text { Sulla and wheat, surrounded by large } \\
\text { wetlands with characteristic wild } \\
\text { plants }\end{array}$ \\
\hline Spina Santa & 18 & {$[A E]$} & $37^{\circ} 24^{\prime} \mathrm{N} 14^{\circ} 57^{\prime} \mathrm{E}$ & Large cultivated areas & $\begin{array}{l}\text { Cereals, sulla, and vegetables, with } \\
\text { natural flora present in the residual } \\
\text { zones }\end{array}$ \\
\hline Motta Sant'Anastasia & 70 & {$[A E]$} & $37^{\circ} 30^{\prime} \mathrm{N} 14^{\circ} 53^{\prime} \mathrm{E}$ & Large cultivated areas & Citrus and little natural vegetation \\
\hline Giarre & 81 & {$[A E]$} & $37^{\circ} 44^{\prime} \mathrm{N} 15^{\circ} 10^{\prime} \mathrm{E}$ & Large cultivated areas & $\begin{array}{l}\text { Avocado, citrus, mango, fejoia, loquat, } \\
\text { and other fruits, with native flora in } \\
\text { marginal zones }\end{array}$ \\
\hline Ramacca & 200 & {$[A E]$} & $37^{\circ} 23^{\prime} \mathrm{N} 14^{\circ} 41^{\prime} \mathrm{E}$ & Agro-ecosystem & $\begin{array}{l}\text { Cereals and oilseeds; marginal areas } \\
\text { with native flora }\end{array}$ \\
\hline Paternò & 256 & {$[A E]$} & $37^{\circ} 33^{\prime} \mathrm{N} 14^{\circ} 54^{\prime} \mathrm{E}$ & Large cultivated areas & $\begin{array}{l}\text { Citrus groves in intensive culture with } \\
\text { residual natural vegetation }\end{array}$ \\
\hline Bronte & 625 & {$[A E]$} & $37^{\circ} 48^{\prime} \mathrm{N} 14^{\circ} 47^{\prime} \mathrm{E}$ & Large cultivated areas & $\begin{array}{l}\text { Peach and pear, surrounded by oak } \\
\text { forests }\end{array}$ \\
\hline Milia & 1046 & {$[S N]$} & $37^{\circ} 39^{\prime} \mathrm{N} 14^{\circ} 59^{\prime} \mathrm{E}$ & Within Etna Park & $\begin{array}{l}\text { Pome and stone-fruit orchards with an } \\
\text { abundance of wild flowers }\end{array}$ \\
\hline Piano Provenzana & 1845 & {$[\mathrm{NE}]$} & $37^{\circ} 47^{\prime} \mathrm{N} 15^{\circ} 02^{\prime} \mathrm{E}$ & $\begin{array}{l}\text { Within Etna Park (site Na- } \\
\text { tura } 2000 \text { ITA } 070009 \text { ) at } \\
\text { the altitudinal limit of pio- } \\
\text { neer vegetation }\end{array}$ & $\begin{array}{l}\text { Rich in plants of high ecological value, } \\
\text { with the presence of many endemic } \\
\text { species }\end{array}$ \\
\hline
\end{tabular}


The species belonged to eleven genera: Amegilla (2 species), Anthophora (6 species), Apis (1 species), Ceratina (7 species), Epeolus (1 species), Eucera (10 species), Habropoda (1 species), Melecta (1 species), Nomada (3 species), Tetraloniella (1 species), and Xylocopa (2 species). Overall, the Apidae genera were represented as follows: Amegilla (12 specimens; $0.08 \%$ of the total number), Anthophora (94 specimens; 0.65\%), Apis (13.666 specimens; 94.57\%) Ceratina (124 specimens; 0.86\%), Epeolus (1 specimen; 0.01\%), Eucera (421 specimens; 2.91\%), Habropoda (13 specimens; 0.09\%), Melecta (1 specimen; $0.01 \%)$, Nomada (18 specimens; 0.12\%), Tetraloniella (3 specimens; 0.02\%), and Xylocopa (98 specimens; 0.68\%) Apis mellifera Linnaeus, was the most numerous and common species, recorded in all environments investigated, due to the widespread practice of beekeeping.

Table 2 shows the recorded entities with the list of plants visited in relation to the altitudinal plans.

\section{FOOD SOURCES}

The surveyed Apidae were observed on 68 species of plants, comprising a total of 63 plant genera within 27 plant families (Tables 2, 3, and Fig. I). Particularly interesting was the site of Piano Provenzana, where there were species

Table 2 - Taxa and related number of specimens of the bees (Apidae family, bumblebees excluded) recorded on Mount Etna in the period 1997-2007 on cultivated and wild plants at different altitudinal plans.

\begin{tabular}{|c|c|c|c|c|c|}
\hline \multicolumn{2}{|c|}{ Bees } & \multicolumn{2}{|r|}{ Plants } & \multirow{2}{*}{ Altitude } & \multirow{2}{*}{$\begin{array}{l}\text { No. } \\
\text { specimens }\end{array}$} \\
\hline Genus & Species/subspecies & Family & Species & & \\
\hline \multirow{3}{*}{ Amegilla } & albigena & Verbenaceae & Vitex agnus-castus & $0-500$ & 5 \\
\hline & \multirow[b]{2}{*}{ quadrifasciata } & Asteraceae, Brassicaceae & Helianthus annuus, Moricandia arvensis & $0-500$ & 4 \\
\hline & & $\begin{array}{c}\text { Boraginaceae, Scrophulariaceae, } \\
\text { Asteraceae }\end{array}$ & $\begin{array}{l}\text { Echium vulgare ssp. pustulatum, Linaria } \\
\text { purpurea,Onopordum illyricum }\end{array}$ & $1000-1450$ & 3 \\
\hline \multirow{8}{*}{ Anthophora } & aestivalis & Asteraceae & Centaurea napifolia & $0-500$ & 1 \\
\hline & balearica & Brassicaceae & Erysimum aetnense & $1450-2100$ & 1 \\
\hline & balneorum & Boraginaceae & Echium vulgare ssp. pustulatum & $1000-1450$ & 1 \\
\hline & crinipes & Asteraceae & Onopordum illyricum & $1000-1450$ & 10 \\
\hline & \multirow{2}{*}{ plumipes squalens } & Boraginaceae & Echium vulgare ssp. pustulatum & $0-500$ & 67 \\
\hline & & Boraginaceae, Rosaceae & Anchusa hybrida, Pyrus communis & $1000-1450$ & 8 \\
\hline & \multirow{2}{*}{ retusa } & Brassicaceae & Raphanus raphanistrum & $500-1000$ & 2 \\
\hline & & Rosaceae & Rubus aetnicus & $1450-2100$ & 4 \\
\hline \multirow{4}{*}{ Apis } & \multirow{4}{*}{ mellifera } & $\begin{array}{c}\text { Apiaceae, Asteraceae, } \\
\text { Boraginaceae, Brassicaceae, } \\
\text { Convolvulaceae, Cucurbitaceae, } \\
\text { Dipsacaceae, Euphorbiaceae, } \\
\text { Fabaceae, Mirtaceae, Oxalidaceae, } \\
\text { Resedaceae, Rosaceae, Rutaceae, } \\
\text { Tamaricaceae, Verbenaceae }\end{array}$ & $\begin{array}{c}\text { Ammi visnaga, Anthemis arvensis, } \\
\text { Borago officinalis, Brassica nigra, } \\
\text { Calendula arvensis, Centaurea napifolia, } \\
\text { Cerinthe major, Chrozophora tinctoria, } \\
\text { Citrullus lanatus, Citrus sinensis, } \\
\text { Calystegia sepium, Diplotaxis erucoides, } \\
\text { Diplotaxis tenuifolia, Dittrichia viscosa, } \\
\text { Echium vulgare ssp. pustulatum, } \\
\text { Eriobotrya japonica, Eucalyptus camaldulensis, } \\
\text { Galactites tomentosa, Hedysarum coronarium, } \\
\text { Helianthus annuus, Heliotropium europaeum, } \\
\text { Knautia integrifolia, Melilotus officinalis, } \\
\text { Oxalis pes-caprae, Reseda alba, } \\
\text { Rubus ulmifolius, Scabiosa maritima, } \\
\text { Sonchus oleraceus, Tamarix gallica, } \\
\text { Vitex agnus-castus }\end{array}$ & $0-500$ & 10.737 \\
\hline & & $\begin{array}{l}\text { Asteraceae, Brassicaceae, } \\
\text { Onagraceae, Rosaceae }\end{array}$ & $\begin{array}{l}\text { Diplotaxis erucoides, Dittrichia viscosa, } \\
\text { Epilobium hirsutum, Malus domestica, } \\
\text { Prunus spinosa, Pulicaria dysenterica, } \\
\text { Pyrus communis, Rubus ulmifolius }\end{array}$ & $500-1000$ & 400 \\
\hline & & $\begin{array}{c}\text { Asteraceae, Fagaceae, } \\
\text { Geraniaceae, Labiatae, Lamiaceae, } \\
\text { Rosaceae, Scrophulariaceae }\end{array}$ & $\begin{array}{l}\text { Amygdalus communis, Calamintha foliosa, } \\
\text { Carlina nebrodensis, Castanea sativa, } \\
\text { Erodium acaule, Linaria purpurea, } \\
\text { Malus domestica, Micromeria graeca, } \\
\text { Onopordum illyricum, Pyrus communis, } \\
\text { Salvia verbenaca, Satureja fruticosa, } \\
\text { Senecio glaber, Rubus ulmifolius }\end{array}$ & $1000-1450$ & 448 \\
\hline & & $\begin{array}{c}\text { Asteraceae, Berberidaceae, } \\
\text { Fabaceae, Onagraceae, Rosaceae, } \\
\text { Rubiaceae, Scrophulariaceae }\end{array}$ & $\begin{array}{c}\text { Astragalus siculus, Berberis aetnensis, } \\
\text { Epilobium angustifolium, Galium aetnicum, } \\
\text { Linaria purpurea, Rosa canina, Rubus aetnicus, } \\
\text { Senecio aetnensis, Tanacetum siculum }\end{array}$ & $1450-2100$ & 2.081 \\
\hline \multirow{3}{*}{ Ceratina } & \multirow{2}{*}{ chalcites } & Boraginaceae & Echium vulgare ssp. pustulatum & $500-1000$ & 2 \\
\hline & & Asteraceae & Onopordum illyricum & $1000-1450$ & 1 \\
\hline & chalybea & Asteraceae & Senecio aetnensis, Tanacetum siculum & $1450-2100$ & 7 \\
\hline
\end{tabular}


Continued Table 2

\begin{tabular}{|c|c|c|c|c|c|}
\hline \multicolumn{2}{|c|}{ Bees } & \multicolumn{2}{|r|}{ Plants } & \multirow{2}{*}{ Altitude } & \multirow{2}{*}{$\begin{array}{l}\text { No. } \\
\text { specimens }\end{array}$} \\
\hline Genus & Species/subspecies & Family & Species & & \\
\hline \multirow{8}{*}{ Ceratina } & \multirow{3}{*}{ cucurbitina } & Boraginaceae, Verbenaceae & $\begin{array}{l}\text { Echium vulgare ssp. pustulatum, } \\
\text { Vitex agnus-castus }\end{array}$ & $0-500$ & 9 \\
\hline & & $\begin{array}{l}\text { Asteraceae, Boraginaceae, } \\
\text { Brassicaceae, Rosaceae }\end{array}$ & $\begin{array}{c}\text { Diplotaxis erucoides, Echium vulgare ssp. } \\
\text { pustulatum, Galactites tomentosa, } \\
\text { Malus domestica }\end{array}$ & $500-1000$ & 15 \\
\hline & & $\begin{array}{l}\text { Asteraceae, Boraginaceae, } \\
\text { Rosaceae, Scrophulariaceae }\end{array}$ & $\begin{array}{c}\text { Bellis perennis, Echium vulgare ssp. pustulatum, } \\
\text { Linaria purpurea, Malus domestica, } \\
\text { Pyrus communis, Senecio glaber }\end{array}$ & $1000-1450$ & 65 \\
\hline & cyanea & Asteraceae, Verbenaceae & Dittrichia viscosa, Vitex agnus-castus & $0-500$ & 15 \\
\hline & dallatorreana & $\begin{array}{l}\text { Asteraceae, Boraginaceae, } \\
\text { Verbenaceae }\end{array}$ & $\begin{array}{l}\text { Echium vulgare ssp. pustulatum, } \\
\text { Helianthus annuus, Vitex agnus-castus }\end{array}$ & $0-500$ & 7 \\
\hline & \multirow{2}{*}{ dentiventris } & Boraginaceae & Echium vulgare ssp. pustulatum & $500-1000$ & 1 \\
\hline & & Boraginaceae & Heliotropium europaeum & $1000-1450$ & 1 \\
\hline & nigrolabiata & Boraginaceae & Echium vulgare ssp. pustulatum & $500-1000$ & 1 \\
\hline Epeolus & julliani & Scrophulariaceae & Linaria purpurea & $1450-2100$ & 1 \\
\hline \multirow{15}{*}{ Eucera } & aterrima & Fabaceae, Rosaceae & Astragalus siculus, Rubus aetnicus & $1450-2100$ & 7 \\
\hline & caspica & Boraginaceae, Fabaceae & Anchusa hybrida, Vicia sativa & $1000-1450$ & 3 \\
\hline & \multirow[t]{2}{*}{ eucnemidea } & $\begin{array}{l}\text { Asteraceae, Boraginaceae, } \\
\text { Fabaceae, Malvaceae }\end{array}$ & $\begin{array}{c}\text { Centaurea napifolia, Echium vulgare ssp. } \\
\text { pustulatum, Galactites tomentosa, Glebionis } \\
\text { coronaria, Hedysarum coronarium, Lavatera } \\
\text { trimestris, Reichardia picroides }\end{array}$ & $0-500$ & 364 \\
\hline & & Asteraceae & Galactites tomentosa & $500-1000$ & 2 \\
\hline & \multirow{3}{*}{ nigrescens } & Fabaceae & Hedysarum coronarium & $0-500$ & 1 \\
\hline & & Boraginaceae, Fabaceae & Borago officinalis, Vicia sativa & $500-1000$ & 6 \\
\hline & & Fabaceae & Vicia sativa & $1000-1450$ & 1 \\
\hline & \multirow{2}{*}{ notata } & Boraginaceae & Echium vulgare ssp. pustulatum & $0-500$ & 3 \\
\hline & & Asteraceae, Fabaceae & Lathyrus grandiflorus, Onopordum illyricum & $1000-1450$ & 5 \\
\hline & numida numida & Fabaceae & Hedysarum coronarium & $0-500$ & 2 \\
\hline & \multirow{2}{*}{ oraniensis } & Asteraceae & Galactites tomentosa & $0-500$ & 9 \\
\hline & & Rosaceae & Amygdalus communis & $1000-1450$ & 5 \\
\hline & proxima & Fabaceae & Hedysarum coronarium & $0-500$ & 3 \\
\hline & seminuda & Asteraceae, Rutaceae & Citrus sinensis, Galactites tomentosa & $0-500$ & 7 \\
\hline & vulpes & Asteraceae, Boraginaceae & $\begin{array}{c}\text { Centaurea napifolia, } \\
\text { Echium vulgare ssp. pustulatum }\end{array}$ & $0-500$ & 3 \\
\hline Habropoda & tarsata & Boraginaceae, Fabaceae, Rosaceae & $\begin{array}{c}\text { Anchusa hybrida, Lathyrus grandiflorus, } \\
\text { Pyrus communis }\end{array}$ & $1000-1450$ & 13 \\
\hline Melecta & albifrons nigra & Boraginaceae & Echium vulgare ssp. pustulatum & $0-500$ & 1 \\
\hline \multirow{3}{*}{ Nomada } & discrepans & Asteraceae, Rosaceae & Pulicaria dysenterica, Rubus ulmifolius & $0-500$ & 9 \\
\hline & distinguenda & Asteraceae, Verbenaceae & Pulicaria dysenterica, Vitex agnus-castus & $0-500$ & 6 \\
\hline & succincta & Rosaceae & Amygdalus communis, Pyrus communis & $1000-1450$ & 3 \\
\hline Tetraloniella & dentata fulviscopa & Asteraceae, Lamiaceae & Marrubium vulgare, Onopordum illyricum & $1000-1450$ & 3 \\
\hline \multirow{6}{*}{ Xylocopa } & \multirow{2}{*}{ iris } & Verbenaceae & Vitex agnus-castus & $0-500$ & 14 \\
\hline & & Lamiaceae, Rosaceae & Amygdalus communis, Micromeria graeca & $1000-1450$ & 2 \\
\hline & \multirow{4}{*}{ violacea } & $\begin{array}{c}\text { Boraginaceae, Rosaceae, } \\
\text { Verbenaceae }\end{array}$ & $\begin{array}{c}\text { Borago officinalis, Eriobotrya japonica, Rubus } \\
\text { ulmifolius, Vitex agnus-castus }\end{array}$ & $0-500$ & 20 \\
\hline & & $\begin{array}{c}\text { Asteraceae, Boraginaceae, } \\
\text { Rosaceae }\end{array}$ & $\begin{array}{c}\text { Borago officinalis, Dittrichia viscosa, Prunus } \\
\text { persica, Pulicaria dysenterica }\end{array}$ & $500-1000$ & 6 \\
\hline & & $\begin{array}{c}\text { Asphodelaceae, Asteraceae, } \\
\text { Boraginaceae, Lamiaceae, } \\
\text { Rosaceae }\end{array}$ & $\begin{array}{c}\text { Amygdalus communis, Anchusa hybrida, } \\
\text { Asphodelus ramosus, Echium vulgare ssp. } \\
\text { pustulatum, Marrubium vulgare, } \\
\text { Pyrus communis, Senecio glaber }\end{array}$ & $1000-1450$ & 41 \\
\hline & & $\begin{array}{l}\text { Caryophyllaceae, Fabaceae, } \\
\text { Rosaceae }\end{array}$ & $\begin{array}{l}\text { Astragalus siculus, Rubus aetnicus, } \\
\text { Saponaria sicula }\end{array}$ & $1450-2100$ & 15 \\
\hline
\end{tabular}


Table 3 - Species of Apidae and botanical families.

\begin{tabular}{|c|c|c|c|c|c|c|c|c|c|c|c|c|c|c|c|c|c|c|c|c|c|c|c|c|c|c|c|}
\hline \begin{tabular}{|}
$\begin{array}{r}\text { Plant's } \\
\text { family }\end{array}$ \\
Apidae \\
species
\end{tabular} & 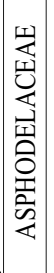 & 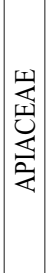 & 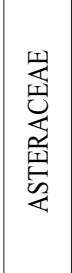 & 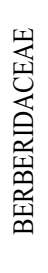 & 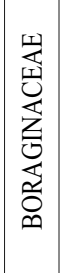 & 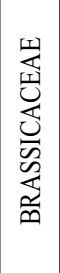 & 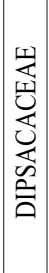 & 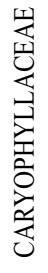 & 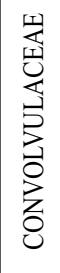 & 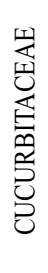 & 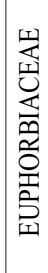 & $\begin{array}{l}\text { 至 } \\
\text { 至 } \\
\text { 产 } \\
\text { 空 }\end{array}$ & 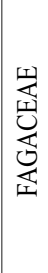 & 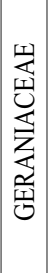 & 岕 & 盛 & 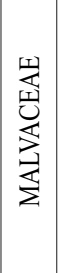 & 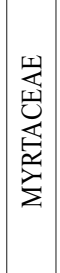 & 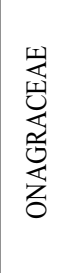 & 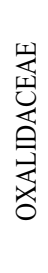 & 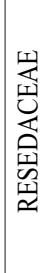 & 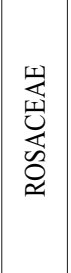 & 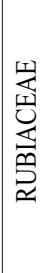 & 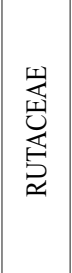 & 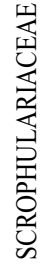 & 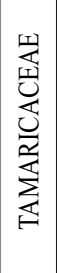 & 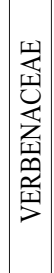 \\
\hline $\begin{array}{l}\text { Amegilla } \\
\text { albigena }\end{array}$ & & & & & & & & & & & & & & & & & & & & & & & & & & & 5 \\
\hline \begin{tabular}{|} 
Amegilla \\
quadrifasciata
\end{tabular} & & & 3 & & 1 & 2 & & & & & & & & & & & & & & & & & & & 1 & & \\
\hline $\begin{array}{l}\text { Anthophora } \\
\text { aestivalis }\end{array}$ & & & 1 & & & & & & & & & & & & & & & & & & & & & & & & \\
\hline $\begin{array}{l}\text { Anthophora } \\
\text { balearica }\end{array}$ & & & & & & 1 & & & & & & & & & & & & & & & & & & & & & \\
\hline $\begin{array}{l}\text { Anthophora } \\
\text { balneorum }\end{array}$ & & & & & 1 & & & & & & & & & & & & & & & & & & & & & & \\
\hline $\begin{array}{l}\text { Anthophora } \\
\text { crinipes }\end{array}$ & & & 10 & & & & & & & & & & & & & & & & & & & & & & & & \\
\hline $\begin{array}{l}\text { Anthophora } \\
\text { plumipes } \\
\text { squalens }\end{array}$ & & & & & 74 & & & & & & & & & & & & & & & & & 1 & & & & & \\
\hline $\begin{array}{l}\text { Anthophora } \\
\text { retusa }\end{array}$ & & & & & & 2 & & & & & & & & & & & & & & & & 4 & & & & & \\
\hline Apis mellifera & & 2 & 1550 & 70 & 878 & 127 & 21 & & 60 & 21 & 2 & 2690 & 50 & 1 & 40 & 100 & & 154 & 386 & 37 & 2 & 4836 & 8 & 2194 & 43 & 365 & 29 \\
\hline $\begin{array}{l}\text { Ceratina } \\
\text { chalcites }\end{array}$ & & & 1 & & 2 & & & & & & & & & & & & & & & & & & & & & & \\
\hline $\begin{array}{l}\text { Ceratina } \\
\text { chalybea }\end{array}$ & & & 7 & & & & & & & & & & & & & & & & & & & & & & & & \\
\hline $\begin{array}{l}\text { Ceratina } \\
\text { cucurbitina }\end{array}$ & & & 8 & & 7 & 1 & & & & & & & & & & & & & & & & 67 & & & 1 & & 5 \\
\hline $\begin{array}{l}\text { Ceratina } \\
\text { cyanea }\end{array}$ & & & 3 & & & & & & & & & & & & & & & & & & & & & & & & 12 \\
\hline $\begin{array}{l}\text { Ceratina } \\
\text { dallatorreana }\end{array}$ & & & 2 & & 1 & & & & & & & & & & & & & & & & & & & & & & 4 \\
\hline \begin{tabular}{|l} 
Ceratina \\
dentiventris
\end{tabular} & & & & & 2 & & & & & & & & & & & & & & & & & & & & & & \\
\hline $\begin{array}{l}\text { Ceratina } \\
\text { nigrolabiata }\end{array}$ & & & & & 1 & & & & & & & & & & & & & & & & & & & & & & \\
\hline $\begin{array}{l}\text { Epeolus } \\
\text { julliani }\end{array}$ & & & & & & & & & & & & & & & & & & & & & & & & & 1 & & \\
\hline $\begin{array}{l}\text { Eucera } \\
\text { aterrima }\end{array}$ & & & & & & & & & & & & 5 & & & & & & & & & & 2 & & & & & \\
\hline $\begin{array}{l}\text { Eucera } \\
\text { caspica }\end{array}$ & & & & & 1 & & & & & & & 2 & & & & & & & & & & & & & & & \\
\hline $\begin{array}{l}\text { Eucera } \\
\text { eucnemidea }\end{array}$ & & & 99 & & 16 & & & & & & & 1 & & & & & 248 & & & & & & & & & & \\
\hline $\begin{array}{l}\text { Eucera } \\
\text { nigrescens }\end{array}$ & & & & & 2 & & & & & & & 6 & & & & & & & & & & & & & & & \\
\hline Eucera notata & & & 2 & & 3 & & & & & & & 3 & & & & & & & & & & & & & & & \\
\hline $\begin{array}{l}\text { Eucera } \\
\text { numida } \\
\text { numida }\end{array}$ & & & & & & & & & & & & 2 & & & & & & & & & & & & & & & \\
\hline $\begin{array}{l}\text { Eucera } \\
\text { oraniensis }\end{array}$ & & & 9 & & & & & & & & & & & & & & & & & & & 5 & & & & & \\
\hline \begin{tabular}{|l} 
Eucera \\
proxima
\end{tabular} & & & & & & & & & & & & 3 & & & & & & & & & & & & & & & \\
\hline $\begin{array}{l}\text { Eucera } \\
\text { seminuda }\end{array}$ & & & 6 & & & & & & & & & & & & & & & & & & & & & 1 & & & \\
\hline Eucera vulpes & & & 2 & & 1 & & & & & & & & & & & & & & & & & & & & & & \\
\hline $\begin{array}{l}\text { Habropoda } \\
\text { tarsata }\end{array}$ & & & & & 5 & & & & & & & 7 & & & & & & & & & & 1 & & & & & \\
\hline
\end{tabular}


Continued Table 3

\begin{tabular}{|c|c|c|c|c|c|c|c|c|c|c|c|c|c|c|c|c|c|c|c|c|c|c|c|c|c|c|c|}
\hline $\begin{array}{r}\begin{array}{r}\text { Plant's } \\
\text { family }\end{array} \\
\text { Apidae } \\
\text { species }\end{array}$ & 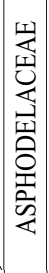 & 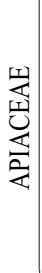 & 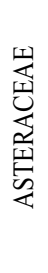 & 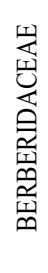 & 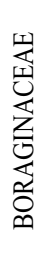 & 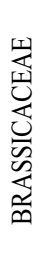 & 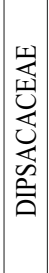 & 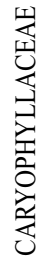 & 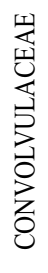 & 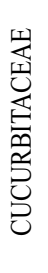 & 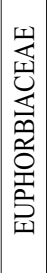 & 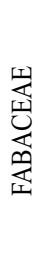 & $\begin{array}{l}\text { 至 } \\
\text { 空 } \\
\text { 至 }\end{array}$ & 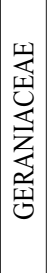 & 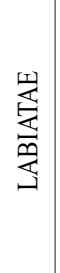 & $\begin{array}{l}\text { 至 } \\
\text { 恶 } \\
\sum_{\Xi}\end{array}$ & 焉 & 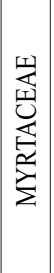 & 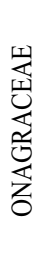 & 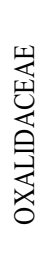 & 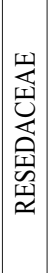 & 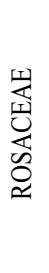 & 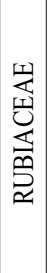 & $\begin{array}{l}\text { 至 } \\
\text { 至 } \\
\text { 岕 } \\
\text { 总 }\end{array}$ & 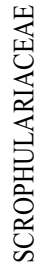 & 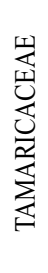 & 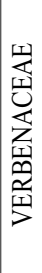 \\
\hline $\begin{array}{l}\text { Melecta } \\
\text { albifrons nigra }\end{array}$ & & & & & 1 & & & & & & & & & & & & & & & & & & & & & & \\
\hline $\begin{array}{l}\text { Nomada } \\
\text { discrepans }\end{array}$ & & & 6 & & & & & & & & & & & & & & & & & & & 3 & & & & & \\
\hline $\begin{array}{l}\text { Nomada } \\
\text { distinguenda }\end{array}$ & & & 3 & & & & & & & & & & & & & & & & & & & & & & & & 3 \\
\hline $\begin{array}{l}\text { Nomada } \\
\text { succincta }\end{array}$ & & & & & & & & & & & & & & & & & & & & & & 3 & & & & & \\
\hline $\begin{array}{l}\text { Tetraloniella } \\
\text { dentata } \\
\text { fulviscopa }\end{array}$ & & & 2 & & & & & & & & & & & & & 1 & & & & & & & & & & & \\
\hline Xylocopa iris & & & & & & & & & & & & & & & & 1 & & & & & & 1 & & & & & 14 \\
\hline $\begin{array}{l}\text { Xylocopa } \\
\text { violacea }\end{array}$ & 1 & & 3 & & 4 & & & 6 & & & & 7 & & & & 4 & & & & & & 45 & & & & & 12 \\
\hline
\end{tabular}

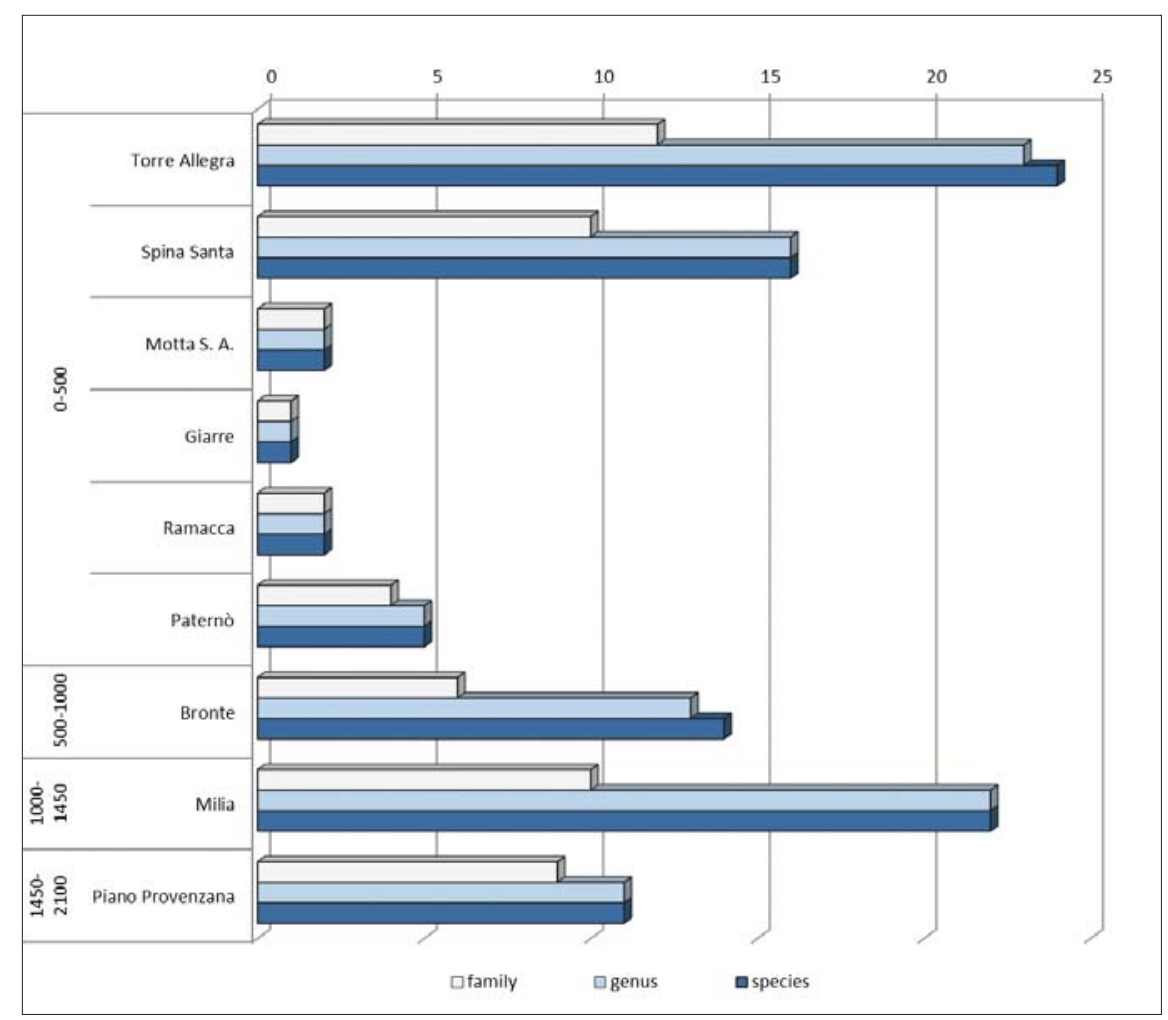

Fig. I - Number of families, genera, and species of plants visited by bees in the period 1997-2007 in relation to the altitudinal plans.

of considerable phytogeographic importance, such as Astragalus siculus (Fabaceae) and Senecio aethnensis (Asteraceae), characteristic of high altitudes of Etna, and Erysimum aetnense Jord. (Brassicaceae), all endemic to Sicily. In this environment, most of the bees were recorded on plants with the highest potential of honey, such as Astragalus, Rubus and Senecio. Here, despite the position inside a protected area, the total number of plants was low (Fig. I) due to the extreme climatic conditions of this high mountain environment. The largest number of plant species was found in the two semi-natural ecosystems of Torre Allegra and Milia and in the agro-ecosystem of Bronte (Fig. I). In this latter, this is explained with the presence of a large number of wild plants surrounding the agro-ecosystems. The herbaceous and woody cultivated plants visited by bees included 11 species within Castanea, Citrus, Eucalyptus, Malus, Prunus, and Pyrus (spring flowering); Hedysarum and Helianthus (summer flowering); and Amygdalus and Eriobotrya (winter flowering). Only five species of bees were found on almond trees (cultivated at an altitude of 
$1000 \mathrm{~m})$ and loquat $(81 \mathrm{~m})$ in December-January and December-February, respectively (Table 2). In this adverse season, only the following species were noticed: Apis mellifera, Eucera oraniensis Lepeletier, Nomada succincta Panzer, Xylocopa iris (Christ) and X. violacea (L.).

Table 3 shows the number of species of bees observed on each botanical family of plants surveyed. Most of the species of the bees were found on Asteraceae (19 species), Rosaceae (11 species), and Fabaceae (10 species). As regards the food preferences, only a few bee species visited a large number of plants belonging to different families (polylectic bees, e.g. Apis mellifera, Ceratina cucurbitina and Xylocopa violacea), whereas the majority were attracted by only two or three botanical species (oligolectic bees) (Table 3).

\section{DETECTED TAXA}

Family Apidae Latreille, 1802

Genus Amegilla Friese, 1897

Amegilla (Zebramegilla) albigena (Lepeletier, 1841) Distribution: Central-Asiatic, European-Mediterranean. Examined specimens: Torre Allegra, 28.VII.1998, 1 ô, (3), 23.VII.1999, 1 § , SB leg., MQ det.

\section{Amegilla (Amegilla) quadrifasciata}

(De Villers, 1789)

Distribution: Palaearctic.

Examined specimens: Ramacca, 23.VI.1999, 1 , 02.VII.1999, 2 우, 12.VII.1999, 1 ㅅ, SB leg., MQ det.; Milia, 21.IX.2004, (1), 16.VII.2005, 19, (1), SB leg. and det.

Genus Anthophora Latreille, 1803

Anthophora (Anthophora) crinipes Smith, 1854 (= salviae Panzer, 1804)

Distribution: Asiatic-European.

Examined specimens: Milia, 07.V.2003, 1 ô, (9), SB leg., MQ det.

\section{Anthophora (Anthophora) plumipes squalens} Dours, 1869

Distribution: South-central Europe, and Maghreb. Examined specimens: Torre Allegra, 14.IV.1998, 5 q 9,1 o, (12), 22.IV.1998, 5 우, (28), VL leg., 06.V.1998, 5 우, (10), 04.V.1999, 1 ㅇ, SB leg., MQ det.; Milia, 24.III.2000, 1 ふ̃ , (6), 7.V.2003, 1 क, SB leg., MQ det.

Anthophora (Paramegilla) balneorum (Lepeletier, 1841) Distribution: South-West Mediterranean.

Examined specimens: Milia, 30.V.2006, 1 đ̊, SB leg., MQ det.

Anthophora (Pyganthophora) aestivalis (Panzer, 1801) Distribution: Europe, Anatolia, and Caucasia.

Examined specimens: Torre Allegra, 26.V.1999, 1 q, SB leg., MQ det.

Anthophora (Pyganthophora) balearica (Friese, 1896) Distribution: Spain and Baleari Islands, southern Italy (Apulia and Sicily), and Anatolia.

Examined specimens: Piano Provenzana, 19.VI.2007, 1 ô, AS \& SB leg., VN det.

Remarks: MAIDL (1922) reported this species for Spain, Italy and Anatolia.

Anthophora (Pyganthophora) retusa (Linnaeus, 1758) Distribution: Palaearctic.

Examined specimens: Bronte, 13.IV.2000, 1 ๙ , (1), SB leg., MQ det.; Piano Provenzana, 27.VII.2007, 1 \%, (3), AS \& SB leg., VN det.
Genus Apis Linnaeus, 1758

Apis mellifera Linnaeus, 1758

Distribution: Cosmopolitan.

Examined specimens: abundant in all sites surveyed (Table 2).

Remark: in relation to the widespread presence in the island territory of beekeeping activity, the specimens observed were mainly related to the two subspecies present in Sicily (Apis mellifera siciliana Dalla Torre, 1896 and $A$. m. ligustica Spinola, 1806) and their hybrids.

Genus Ceratina Latreille, 1802

Ceratina (Ceratina) cucurbitina (Rossi, 1792)

Distribution: W-Palaearctic.

Examined specimens: Torre Allegra, 28.VIII.1997, 1 ㅇ, (2), VL leg., 04.V.1999, 1 q, (3), 28.VI.1999, 2 우, SB leg., MQ det.; Bronte, 30.VI.2000, 1 \&, SB leg., MQ det., 27.IV.2005, 1 ô, 2 o , , (9), 29.V.2006, 2 우, SB leg., VN

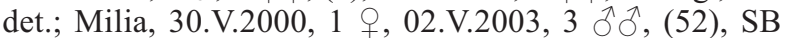
leg., MQ det., 23.IV.2005, 2 $\hat{\sigma}^{\hat{\sigma}}$, (4), 24.IX.2005, 1 \%, 11.IV.2006, 1 §., 30.V.2006, 1 ふे., SB leg., VN det.

Ceratina (Euceratina) chalcites Germar, 1839

Distribution: Europe and northern Asia (excluding China). Examined specimens: Bronte, 19.VII.2005, 1 \% , (1), SB leg., VN det.; Milia, 23.VI.2006, 1 q, SB leg., VN det.

Ceratina (Euceratina) chalybea Chevrier, 1872

Distribution: W-Palaearctic.

Examined specimens: Piano Provenzana, 16.VI.2007, 1 đ̊, (4), 16.VIII.2007, 1 §, 28.VIII.2007, 1 q, AS \& SB leg., VN det.

Ceratina (Euceratina) cyanea (Kirby, 1802)

Distribution: European.

Examined specimens: Spina Santa, 27.X.1998, 1 đ̊ (2), SB leg., MQ det.; Torre Allegra, 28.VI.1999, 1 ô, 23.VII.1999, 2 ơ, (9), SB leg., MQ det.

Ceratina (Euceratina) dallatorreana Friese, 1896 Distribution: Holartic.

Examined specimens: Torre Allegra, 04.V.1999, 1 ㅇ, 28.VI.1999, 1 q, 1 ふै, (2), SB leg., VN det.; Ramacca, 02.VII.1999, 2 우, SB leg., MQ det.

Ceratina (Euceratina) dentiventris Gerstaecker, 1869

Distribution: Caucasia, southern Europe, Anatolia and the Maghreb.

Examined specimens: Bronte, 30.VI.2000, 1 ๙ૈ, SB leg., MQ det.; Milia, 20.VIII 2005, 1 §ै, SB leg., VN det.

Ceratina (Euceratina) nigrolabiata Friese, 1896 Distribution: Europe and northern Asia (excluding China). Examined specimens: Bronte, 29.V.2006, 1 q, SB leg., MQ det.

Genus Epeolus Latreille, 1802

Epeolus julliani Peréz, 1884

Distribution: Southern Europe and Anatolia.

Examined specimens: Piano Provenzana, 16.VIII.2007, 1 +, AS \& SB leg., VN det.

Genus Eucera Scopoli, 1770

Eucera (Atopeucera) seminuda Brullé, 1832 Distribution: Medium-South Europe, Anatolia and Maghreb. Examined specimens: Spina Santa, 25.IV.1998, 2 우, (4), SB leg., MQ det.; Motta Sant'Anastasia, 10.V.2000, 2 우, SB leg., MQ det. 
Eucera (Eucera) proxima Morawitz, 1875 (= graeca Radoszkowski, 1875)

Distribution: South-central Europe, Anatolia, and eastern Mediterranean.

Examined specimens: Spina Santa, 16.IV.1998, 1 ô, (2), SB leg., MQ det.

Eucera (Eucera) nigrescens Pérez, 1879

Distribution: Europe and Anatolia.

Examined specimens: Torre Allegra, 14.IV.1998, 1 ㅇ, SB leg., MQ det.; Bronte, 13.IV.2000, 1 §, 1 O, 26.IV.2000, 1

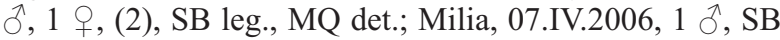
leg., MQ det.

\section{Eucera (Eucera) numida numida} Lepeletier, 1841

Distribution: South-West-Europe and Maghreb. Examined specimens: Torre Allegra, 06.V.1998, 2 + 9 , SB leg., MQ det.

\section{Eucera (Hetereucera) notata Lepeletier, 1841}

Distribution: Mediterranean.

Examined specimens: Paternò, 27.IV.1998, 1 ㅇ, (2), SB leg., MQ det.; Milia, 29.III.2000, 1 गे (2), 27.VI.2000, (2), SB leg.. MQ det.

\section{Eucera (Hetereucera) oraniensis}

Lepeletier, 1841

Distribution: South-West Europe, and northern African. Examined specimens: Torre Allegra, 14.IV.1998, 1 q, (2), SB leg., MQ det.; Spina Santa, 23.III.1998, 1 q, (2), 16.IV.1998, 1 \%, (2), SB leg., MQ det.; Milia, 26.III.2003, 1 ô (4), SB leg., MQ det.

Eucera (? Pareucera) aterrima Friese, 1896 Distribution: Mediterranean. Examined specimens: Piano Provenzana, 02.VII.2006, 1 o, 16.VII.2006, 1 ㅇ, (1), 19.VI.2007, 1 o , 27.VII.2007, 1 , , (2), AS \& SB leg., VN det..

Remark: in this context and until the males are identified, we prefer to believe that Eucera aterrima is a good species and not a subspecies of E. nigrita Friese 1896, as in RISCH (2001).

Eucera (Pareucera) caspica Morawitz, 1873

Distribution: Central-Asiatic, Europe, and Anatolia. Examined specimens: Milia, 08.IV.2005, 1 Oे, 07.IV.2006, 1 , , (1), SB leg., VN det.

Eucera (Pteneucera) eucnemidea Dours, 1873 Distribution: South-West Europe, and Maghreb. Examined specimens: Torre Allegra, 06.V.1998, 10 ठึ ô, (78), VL leg., MQ det.; Spina Santa, 16.IV.1998, 8 o, (34),

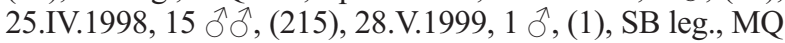
det.; Bronte, 31.V.2000, 1 गे, (1), SB leg., MQ det.

\section{Eucera (Rhyteucera) vulpes Brullé 1832} (= parvula Friese, 1896)

Distribution: South-Europe, and Anatolia. Examined specimens: Torre Allegra, 04.V.1999, 2 우, (1), SB leg., MQ det.

\section{Genus Habropoda Smith, 1854}

Habropoda tarsata (Spinola, 1838)

Distribution: European extended to Anatolia.

Examined specimens: Milia, 17.IV.2000, 1 ภे (4), 22.IV.2000, 1 o, (1), SB leg., MQ det., 07.V.2003, 1 o (5), SB leg., VN det.
Genus Melecta Latreille, 1802

Melecta albifrons nigra Spinola, 1806

Distribution: South-West Europe.

Examined specimens: Torre Allegra, 14.IV.1998, 1 q, SB leg., MQ det.

\section{Genus Nomada Scopoli, 1770}

Nomada discrepans Schmiedeknecht, 1882

Distribution: South-Europe, Maghreb, and Macaronesia. Examined specimens: Torre Allegra, 04.VIII.1997, 1 ô, (2), 28.VIII.1997, 1 ふे, (5), VL leg., MQ det.

Nomada distinguenda Morawitz, 1874

Distribution: Palaearctic.

Examined specimens: Torre Allegra, 04.VIII.1997, 1 ô, (2), 28.VIII.1997, 1 ふै, (2), VL leg., MS det.

Nomada succincta Panzer, 1798

Distribution: Asiatic-European.

Examined specimens: Milia, 29.III.2000, 1 q, 31.III.2006, 1 §, 04.IV.2006, 1 ㅇ, SB leg., MQ det.

Genus Tetraloniella Ashmead, 1899

Tetraloniella dentata fulviscopa Hedicke, 1933

Distribution: South-Europe, and Caucasia.

Examined specimens: Milia, 22.VI.2005, 1 §, 23.VI.2006, $2 \hat{\jmath} \hat{\jmath}, \mathrm{SB}$ leg., $\mathrm{VN}$ det.

Genus Xylocopa Latreille, 1802

Xylocopa (Copoxyla) iris (Christ, 1791)

Distribution: Mediterranean and Central-Asiatic.

Examined specimens: Torre Allegra, 04.VIII.1997, 1 ㅇ, (2), 27.IX.1997, 1 ô, (2), VL leg., 28.VII.1998, 1 ô, (1), 28.VI.1999, 1 ô, 23.VII.1999, 1 ô, 1 ㅇ, (3), SB leg., MQ det.; Milia, 13.III.2003, 1 §, VN det., 16.VII.2005, (1), SB leg. and det.

Xylocopa (Xylocopa) violacea (Linnaeus, 1758)

Distribution: Europe extended to the Maghreb and Anatolia. Examined specimens: Torre Allegra, 28.VIII.1997, $3 \hat{\jmath} \hat{\jmath}, 1$

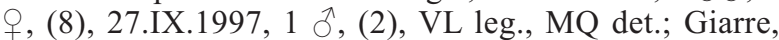
14.XI.2000, (2); Paternò, 27.IV.1998, 1 ô, (2), SB leg., MQ det.; Bronte, 07.IV.2005, (1), 27.IV.2005, (3), 26.IX.2005, (1), 28.IX.2006, (1); Milia, 24.III.2000, 2 ๙ึَ, (1), 20.IV.2000, 1 9, 5.III.2003, 1 ठे, (4), 13.III.2003, 1 के, (29), 19.III.2003, 1 o, (1), SB leg., MQ det.; Piano Provenzana, 19.VIII.2006, 1 गे, (14), AS \& SB leg., VN det.

\section{DISCUSSION AND CONCLUSIONS}

In the 11 years of investigation, more than 20000 specimens of Apoidea were collected and/or observed, more than 18000 of which belonged to the family Apidae, accounting for $88 \%$ of the total. Excluding bumblebees that accounted for 3426 specimens in 12 species (MAZZEO et al., 2015), 14451 Apidae specimens in 11 genera and 35 species were detected. Overall, the Apidae family (Bombus genus included) was present with 48 taxa.

Apis mellifera, with 13666 specimens was the predominant species for number and range, having been found on Mount Etna at all altitudes. Xylocopa violacea was also very common and widely distributed. Only seven species were recorded for the site at the highest altitude, Piano Provenzana (1845 m a.s.1.): Anthophora balearica, A. retusa, Apis mellifera, Ceratina chalybea, Epeolus julliani, Eucera aterrima and Xylocopa violacea. The greatest number of species was found in the thermo-Mediterranean (0-500 $\mathrm{m}$ a.s.1.) and mesoMediterranean (500-1000 m a.s.l.) horizons (Fig. II), where the 


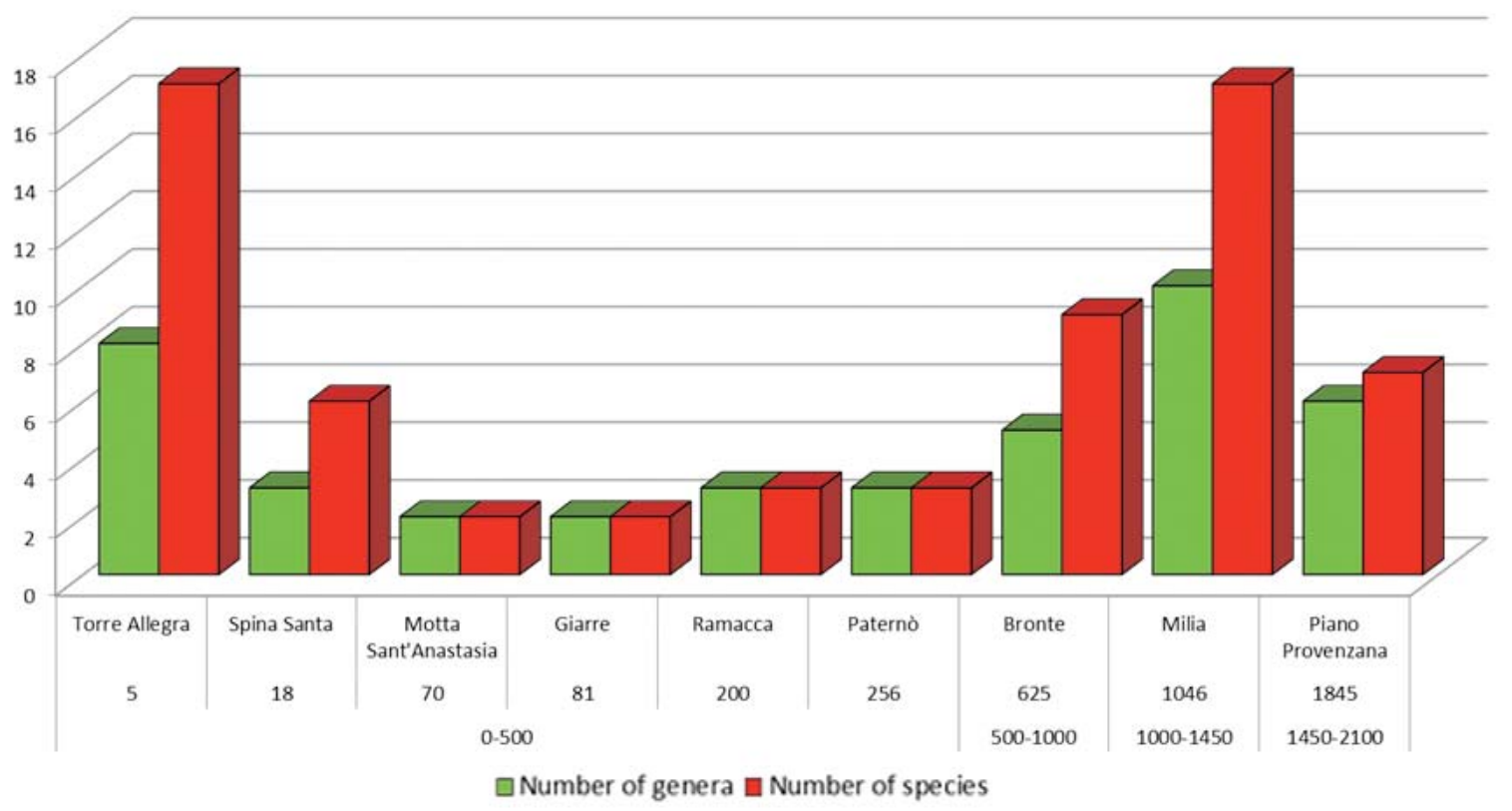

Fig. II - Number of Apidae genera and species (bumblebees excluded) per site recorded on cultivated and wild plants at different altitudinal plans.

presence of bees was abundant even in the coldest months of the year on winter-flowering crops, as in the case of almond (5 species) and loquat (2 species). In this horizons Anthophora plumipes, Apis mellifera and Eucera eucnemidea, were the most abundant species. In the mountain-mediterranean (1450$2100 \mathrm{~m}$ a.s.l.) plan and in the supra-mediterranean horizon (1000-1450 m a.s.1.), Apis mellifera and Xylocopa violacea were most abundant.

Anthophora aestivalis, A. balearica, A. balneorum, Ceratina chalcites, $C$. dentiventris, $C$. nigrolabiata, Epeolus julliani, Eucera proxima, E. numida, E. parvula, Melecta albifrons nigra, Nomada succincta and Tetraloniella dentata were very rare in the period of investigation, with only 1-3 specimens recorded for each species.

Most of the species were recorded inside the protected areas and in particular: Amegilla albigena, Anthophora aestivalis, Apis mellifera, Ceratina cucurbitina, C. cyanea, C. dallatorreana, Eucera nigrescens, E. numida, E. oraniensis, E. eucnemidea, E. vulpes, Melecta nigra, Nomada discrepans, $N$. distinguenda, Xylocopa iris and $X$. violacea from Torre Allegra; Amegilla quadrifasciata, Anthophora crinipes, Anthophora plumipes squalens, Anthophora balneorum, Anthophora balearica, Anthophora retusa, Apis mellifera, Ceratina cucurbitina, $C$. chalcites, C. chalybea, $C$. dentiventris, Epeolus julliani, Eucera nigrescens, E. notata, E. oraniensis, E. aterrima, E. caspica, Habropoda tarsata, Nomada succincta, Tetraloniella dentata fulviscopa, Xylocopa iris, and $X$. violacea from the Etna Park.

The qualitative and quantitative characterisations of the pollinators of a given area provide the "health status" of that territory and, through subsequent monitoring, show the environmental changes that have occurred and how much these have affected biodiversity.

The data from this study, together with those about the Bombus species in MAzzEO et al. (2015), analysed the biodiversity of the Apidae family in the cultivated, seminatural, and natural ecosystems of the Etna territory, a wide area of eastern Sicily, placing it in relation to the different human activities, altitudinal plans, plants visited, and ecological qualities of the areas.

\section{ACKNOWLEDGEMENTS}

We express our gratitude to Dr Vittorio Nobile (Ragusa, Italy) and to Dr Marino Quaranta (CREA-AA, Bologna, Italy) for identifying most of the Apidae collected, and to Dr Giovanna Ferrauto and to prof. Gianpietro Giusso del Galdo (University of Catania, Italy) for the nomenclatural information on the reported plants, and to the anonymous reviewers for their constructive commentary. We are especially grateful to Etna Park and the Metropolitan City of Catania for authorising the surveys in the Regional protected areas.

\section{REFERENCES}

Balzan M.V., Rasmont P., Kuhlmann M., Dathe H., Pauly A., Patiny S., Terzo M., Michez D., 2016 - The bees (Hymenoptera: Apoidea) of the Maltese Islands. Zootaxa, 4162 (2): 225-244.

BeLla S., 2014 - Invasive insect pests and their associated parasitoids on ornamental urban plants on Corfu island Phytoliriomyza jacarandae Steyskal and Spencer 1978 (Diptera, Agromyzidae) a new record in Greece. Hellenic Plant Protection Journal, 7: 53-59.

Celli G., Maccagnani B., 2003 - Honey bees as bioindicators of environmental pollution. - Bulletin of Insectology, 56 (1): 137-139.

Comba M., 2015 - Hymenoptera: Apoidea: Anthophila of Italy. Available from: http: //digilander.libero.it/mario. comba [accessed 07 March, 2019].

Kuhlmann M., Ascher J.S., Dathe H.H., Ebmer A.W., Hartmann P., Michez D., Müller A., Patiny S., Pauly A., Praz C., Rasmont P., Risch S., Scheuchl E., 
Schwarz M., Terzo M., Williams P.H., Amiet F., Baldock D., Berg Ø., Bogusch P., Calabuig I., Cederberg B., Gogala A., Gusenleitner F., Josan Z., Madsen H.B., Nilsson A., ØdegaArd F., Ortiz-SÁncheZ J., Paukkunen J., Pawlikowski T., Quaranta M., RoberTS S.P.M., SÁropataKi M., SCHWENNINGER H.-R., Smit J., Söderman G., TOMOzeI B., 2015 - Checklist of the western Palaearctic bees (Hymenoptera: Apoidea: Anthophila). Available from: http://westpalbees. myspecies.info [accessed 16 July, 2018].

LA GRECA M., 1964 - Le categorie corologiche degli elementi faunistici italiani. - Memorie della Società entomologica italiana, 43: 147-165.

Longo S., Barbagallo S., Rapisarda C., Tropea Garzia G., Mazzeo G., Siscaro G., Bella S., 2001 - Note sull'artropodofauna degli ambienti forestali e agrari del Parco dell'Etna. - Tecnica Agricola, 3-4: 3-61.

MAIDL F., 1922 - Beiträge zur Hymenopterenfauna Dalmatiens, Montenegros und Albaniens. I. Teil: Aculeata und Ghrysididae. - Annalen des Naturhistorischen Museums in Wien, 35: 36-106.

Margiotta M., Bella S., Buffa F., Caleca V., Floris I., Giorno V., Lo Verde G., Rapisarda C., Sasso R., Suma P., Tortorici F., Laudonia S., 2017 - Modeling environmental influences in the Psyllaephagus bliteus (Hymenoptera: Encyrtidae)-Glycaspis brimblecombei (Hemiptera: Aphalaridae) parasitoid-host system. Journal of Economic Entomology, 110 (2): 491-501.

Mazzeo G., Bella S., Seminara A.R., Longo S., 2015 Bumblebees in natural and agro-ecosystems at different altitudes from Mount Etna, Sicily (Hymenoptera Apidae Bombinae): long-term faunistic and ecological observations. - Redia, 98: 123-131.

Mazzeo G., Longo S., PALMeri V., 2006 - L'entomofauna antofila indicatrice della biodiversità in ecosistemi naturali e agrari. - Italus Hortus, 13 (2): 266-270.

Mazzeo G., Longo S., Palmeri V., Seminara A.R., Bella S., ZapPALÀ L., 2004 - Anthophilous insects in fruit orchards on mount Etna (Italy). - Redia, 87: 247-251.

Mazzeo G., Longo S., Seminara A.R., Bella S., Ferrauto G., 2007a - Preliminary observations on anthophilous insects and foraging behaviour of Apis mellifera $L$. in a protected area of the Etna Park. - Redia, 90: 109-113.

Mazzeo G., Seminara A.R., Bella S., Ferrauto G., Longo S., 2007b - Osservazioni sugli apoidei e sull'attività di Apis mellifera in un'area altomontana del Parco dell'Etna. - Apoidea, 4: 163-168.

Mazzeo G., Siscaro G., Longo S., 2007c - Gli insetti ausiliari del pero in frutteti dell'Etna. Atti del Convegno: Principali risultati del progetto di ricerca "Miglioramento e valorizzazione delle produzioni frutticole etnee". Catania, 28-29 maggio 2007, Vol. II, 97-116.

Michener C.D., 2007 - The bees of the world. Second edition. John Hopkins University Press; Baltimore, Maryland, 953 pp., 20 pls.

Nieto A., Roberts S.P.M., Kemp J., Rasmont P., Kuhlmann M., Criado M.G., Biesmeijer J.C., Bogusch P., Dathe H.H., La Rúa P.D., De Meulemeester T., Dehon M., Dewulf A., Ortiz-Sánchez F.J., Lhomme P., Pauly A., Potts S.G., Praz C., Quaranta M., Radchenko V.G., Scheuchl E., Smit J., Straka J., Terzo M., Tomozei B., Window J., Michez D. 2014 European Red List of Bees. Publication Office of the European Union; Luxembourg City; $\mathrm{x}+86 \mathrm{pp}$.

PolASZEK A., 2013 - Fauna Europaea: Apidae. In: Mitroiu M.-D. et al., (2013) Fauna Europaea: Hymenoptera Apocryta. Fauna Europaea version 2.6. Available from: http://www.fauna-eu.org [accessed 14 Juny, 2018].

Poli Marchese E., 1991 - Piante e fiori dell'Etna. Sellerio Editore, Palermo, pp. 200.

Poli Marchese E., Patti G., 2000 - Carta della vegetazione dell'Etna. Istituto di Biologia e Ecologia Vegetale, Università degli Studi di Catania.

Porrini C., Ghini S., Girotti S., Sabatini A.G., Gattavecchia E., Celli G., 2002 - Use of honey bees as bioindicators of environmental pollution in Italy. In: Honey Bees: Estimating the Environmental Impact of Chemicals (Devillers J., Pham-Delègue M., Eds), Taylor \& Francis, London and New York, 186-247.

Quaranta M., Ambroselli S., Barro P., Bella S., Carini A., Celli G., Cogoi P., Comba L., Comoli L., Felicioli A., Floris I., InTOPPA F., LONGO S., Maini S., MANino A., Mazzeo G., Medrzycki P., Nardi E., Niccolini L., Palmieri N., Patetta A., Piatti C., Piazza M.G., Pinzauti M., Porporato M., Porrini C., Ricciardelli D'albore G., Romagnoli F., Ruiu L., Satta A., Zandigiacomo P., 2004 - Wild bees in agroecosystems and semi-natural landscapes. 1997-2000 collection period in Italy. - Bulletin of Insectology, 57 (1): 11-61.

Rasmont P., Haubruge E., 2018 - Atlas Hymenoptera. http://www.atlashymenoptera.net. [accessed 21 Oct., 2019].

Risch S., 2001 - Die Arten des Genus Eucera Scopoli 1770 (Hymenoptera, Apidae). Untergattung Pareucera Tkalcü 1979. - Entomofauna, 22 (15), 365-376.

Seminara A.R., Bella S., Mazzeo G., Longo S., 2009 Risultati di un triennio di studi sugli insetti antofili in un biotopo del vulcano Etna. - Apoidea, 6 (2): 118-124.

SIAS, 2014 - Climatologia della Sicilia. Regione Siciliana, Assessorato Agricoltura e Foreste, Gruppo IV - Servizi Allo Sviluppo, Unità di Agrometeorologia - Available from:http://www.sias.regione.sicilia.it/pdf/Climatologia sicilia.pdf. [accessed 04 July, 2014].

STOCн F., 2003 - Checklist of the species of the Italian fauna. On-line version 2.0. Available from: http://www.faunaitalia.it/checklist. [accessed 14 May, 2018]. 